\title{
Comparison of supine and prone miniaturized percutaneous nephrolithotomy in the treatment of lower pole, middle pole and renal pelvic stones: A matched pair analysis
}

Harun Ozdemir ${ }^{1}$, Akif Erbin ${ }^{1}$, Murat Sahan ${ }^{1}$, Metin Savun ${ }^{1}$, Alkan Cubuk ${ }^{1}$, Ozgur Yazici ${ }^{1}$, Mehmet Fatih Akbulut ${ }^{1}$, Omer Sarilar ${ }^{1}$

${ }^{1}$ Department of Urology, Haseki Traning and Research Hospital, Istanbul, Turkey

\section{ABSTRACT}

Purpose: We aimed to compare the outcomes of supine and prone miniaturized percutaneous nephrolithotomy (m-PNL) in the treatment of lower pole, middle pole and renal pelvic stones.

Materials and Methods: 54 patients who performed supine m-PNL between January 2017 and March 2018 and 498 patients who performed prone m-PNL between April 2015 and January 2018 were included in the study. Of the 498 patients, 108 matching 1: 2 in terms of age, gender, body mass index, American Association of Anesthesiology score, stone size, stone localization and hydronephrosis according to the supine m-PNL group were selected as prone $\mathrm{m}-\mathrm{PNL}$ group. The patients with solitary kidney, upper pole stone, urinary system anomaly or skeletal malformation and pediatric patients $(<18$ years old) were excluded from the study. The success was defined as 'complete stone clearance' and was determined according to the $1^{\text {st }}$ month computed tomography.

Results: The operation time and fluoroscopy time in supine m-PNL was significantly shorter than prone $\mathrm{m}-\mathrm{PNL}$ group $(58.1 \pm 45.9$ vs. $80.1 \pm 40.0 \mathrm{~min}$ and $3.0 \pm 1.7 \mathrm{~min}$ vs. $4.9 \pm 4.5 \mathrm{~min}, \mathrm{p}=0.025$ and $\mathrm{p}=0.01$, respectively). When post-operative complications were compared according to the modified Clavien-Dindo classification, overall and subgroup complication rates were comparable between groups. There was no significant difference between the groups in terms of the success rates (supine m-PNL; 72.2\%, prone m-PNL; 71.3\%, $\mathrm{p}=0.902$ ).

Conclusions: Supine m-PNL procedure is more advantageous in terms of operation time and fluoroscopy time in the treatment of lower pole, middle pole and renal pelvic stones.

\section{ARTICLE INFO}

Akif Erbin

http://orcid.org/0000-0001-7147-8288

\section{Keywords:}

Supine Position;

Nephrolithotomy, Percutaneous;

Pelvis

Int Braz J Urol. 2019; 45: 956-64

Submitted for publication:

January 26, 2019

Accepted after revision:

April 01, 2019

Published as Ahead of Print:

June 25, 2019

\section{INTRODUCTION}

The main treatment modalities in urinary system stone disease are extracorporeal shockwave lithotripsy (ESWL), ureterorenoscopy (URS), percutaneous nephrolithotomy (PNL), open and laparoscopic surgery. With the recent advances in technology, endourologic procedures (URS and PNL) among the surgical treatments have gained more popularity. The European Association of Urology (EAU) urolithiasis guideline recommends standard PNL as the first choice in the treatment of kidney stones larger than $2 \mathrm{~cm}$ (1). Although PNL is accepted as a safe method, it can lead to 
life-threatening hemorrhages. Considering that the hemorrhage in standard PNL is directly related to the instruments used, the diameters of the instruments have been reduced over the years. In this context, firstly, the miniaturized PNL (m-PNL) technique was introduced by Jackman et al. in 1988 (2). In the following years, developments have continued with defining smaller diameter systems such as ultra-m-PNL, super $m-P N L$ and micro PNL techniques (3-5). The $\mathrm{m}$-PNL is accepted as the use of $14-22 \mathrm{Fr}$ access sheaths by EAU Urolithiasis Guidelines Panel (6).

In PNL, the original position is accepted as 'prone'. However, PNL can be performed in classic supine or different positions such as Galdacao modification of Valdivia, lateral decubitus, lateral position modification, and reverse lithotomy (7-10). Supine PNL was introduced by Valdivia in 1987 and the first results were reported in 1998 with a series of 557 cases (11, 12). When compared with the prone position, supine position has some advantages such as easier and comfortable patient positioning, possibility of simultaneous retrograde access to kidney, lower renal pelvic pressure and easier intervention to the respiratory tract by the anesthetist (13). There are many studies in literature comparing prone PNL and supine PNL, however, almost all of them are related to standard PNL. In the present study, our purpose was to compare the outcomes of supine and prone m-PNL performed for stones located in lower pole, middle pole and renal pelvis.

\section{MATERIALS AND METHODS}

\section{Study design}

The present study was approved by the Internal Institutional Review Board. Fifty four patients who performed supine m-PNL between January 2017 and March 2018 and 498 patients who performed prone m-PNL between April 2015 and January 2018 were included in the study.

Exclusion criterias were:

- pediatric patients (<8 years old)

- patients with solitary kidney
- patients with kidney stones located in the upper pole

- patients with urinary system anomalies

- patients with skeletal malformations

Of the 498 patients, 108 matching 1:2 in terms of age, gender, body mass index (BMI), American Association of Anesthesiology (ASA) scores, stone size, stone localization and hydronephrosis (HN) according to the supine m-PNL group were selected as prone m-PNL group. Both groups were compared in terms of demographic data (age, gender, BMI, ASA score, previous surgery and ESWL), stone characteristics (size, localization, opacity, hydronephrosis), operative data (side, operation time, fluoroscopy time, number of access, size of access sheath, nephrostomy placement, transfusion, complication) and postoperative data (hospitalization time, hemoglobin drop, transfusion, JJ stent placement, success and complication). Operation time was calculated as the time from the insertion of ureteral catheter to nephrostomy placement. The success was defined as 'complete stone clearance' and was determined according to the $1^{\text {st }}$ month CT. Intraoperative complications were evaluated using the modified Stava classification system; postoperative complications were evaluated according to the modified Clavien-Dindo classification system $(14,15)$.

\section{Preoperative evaluation}

Written and verbal consent was obtained from all patients before the operation. Patient assessment included medical history, physical examination, complete blood count, coagulation tests, serum biochemistry, urinalysis and urine culture. Anticoagulant drugs were discontinued at least 7-10 days week before the operation. All patients were evaluated preoperatively by non-contrast computed tomography (CT). Stone size was determined by measurement of the greatest dimension. In the case of multiple calculi, the sum of the greatest dimension of each stone was calculated. All patients had sterile urine culture prior to surgery. Antibiotic prophylaxis was provided by second generation cephalosporins. The first dose was administered intravenously when anesthesia 
was initiated and the second dose was given 12 hours later.

\section{Supine m-PNL technique}

Following general anesthesia, the patient was placed in the Galdakao-Modified Valdivia position. Under C-arm fluoroscopy guidance, 5 French (Fr) open end ureteral catheter was inserted retrogradely. A Foley catheter was then indwelled and the distal end of the ureteral catheter was fixed on the Foley catheter. Skin surface was marked to indicate the lower rib margin, posterior axillary line and iliac crest (Figure-1). The calyx plane to be punctured was determined by ultrasonography. Retrograde pyelography was done and an 18 gauge percutaneous access needle (Boston Scientific Corporation, Natick MA) was passed into the desired calix under fluoroscopic guidance. A 0.035 inch guidewire (Boston Scientific Corporation, Natick MA) was passed antegradely across the renal pelvis and into the ureter, upper or lower calix. The track was dilated sequentially using fascial and metallic dilators. According to stone sizes, the $15,16.5$ or $21 \mathrm{Fr}$ metallic sheats (Karl Storz, Tutlingen, Germany) were advanced over their metal dilators. A rigid 12Fr nephroscope (Karl Storz, Tuttlingen, Germany) was advanced through the sheath. Stone disintegration was achieved using a Holmium YAG Laser litho- tripter (Sphinx, Lisa laser, USA). Flexible antegrade pyeloureteroscopy was performed if the rigid nephroscope couldn't reach to stone. Stone fragments were removed with basket catheters. At the end of the procedure, retrograde pyelography was done to assess the integrity of the pelvicaliceal system (PCS). If there was no extravasation and irrigant fluid was returning clear, no tube was left (tubeless PNL); otherwise, a nephrostomy tube was left in place.

\section{Prone m-PNL technique}

After the induction of general anesthesia, a 5Fr Ureteral catheter was placed and fixed on the Foley catheter in the lithotomy position. The patient was then repositioned in the prone position. Skin surface was marked to indicate the lower rib margin, posterior axillary line and iliac crest ( $\mathrm{Fi}-$ gure-2). Percutaneous access was achieved under C arm fluoroscopy guidance. The puncture was performed with an 18 gauge percutaneous access needle. Following successful puncture, a 0.035 inch guidewire was advanced through the needle into the PCS or ureter. At later stages, tract dilatation, nephroscopy, stone fragmentation, and stone retrieval were performed in a manner similar to supin $\mathrm{m}-\mathrm{PNL}$. All supine and prone procedures were performed by two experienced urologists at the tertiary referral center.

Figure 1 - Galdakao-Modified Valdivia position in supine m-PNL. The shaded area between lower rib, posterior axillary line and iliac crest shows the subcostal access location.

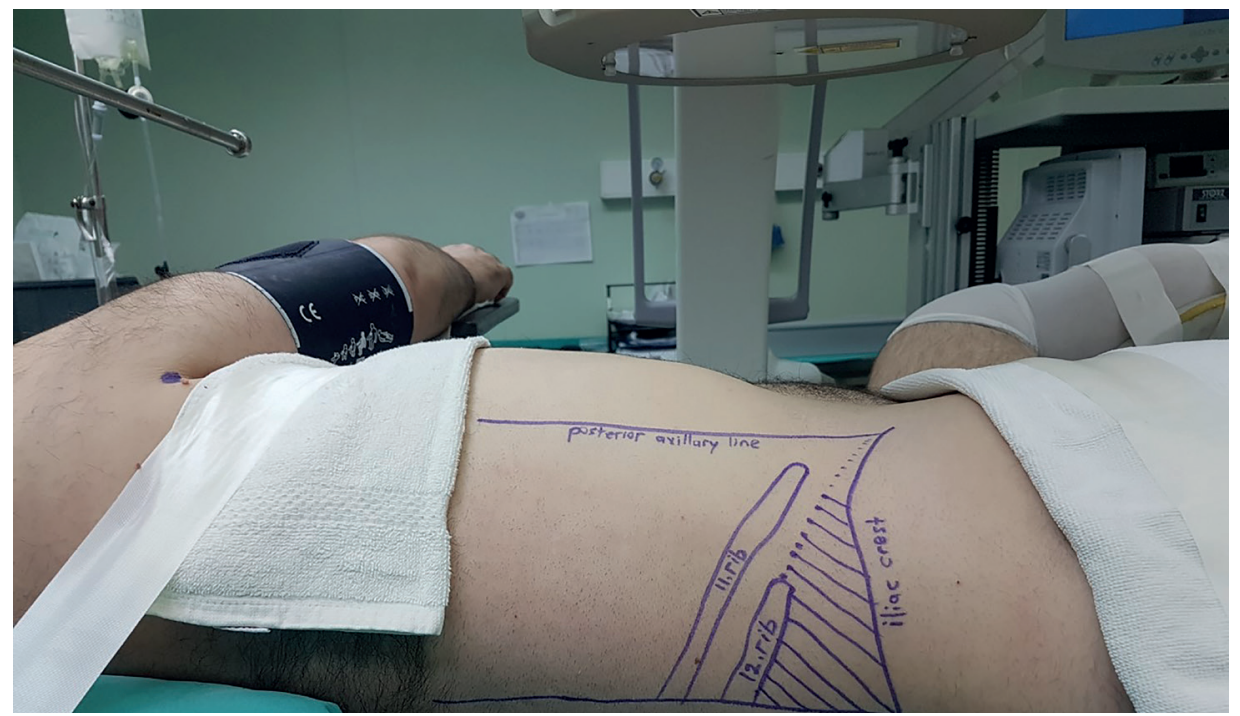


Figure 2 - Prone position. The shaded area between lower rib margin, posterior axillary line and iliac crest shows the subcostal access location in prone PNL.

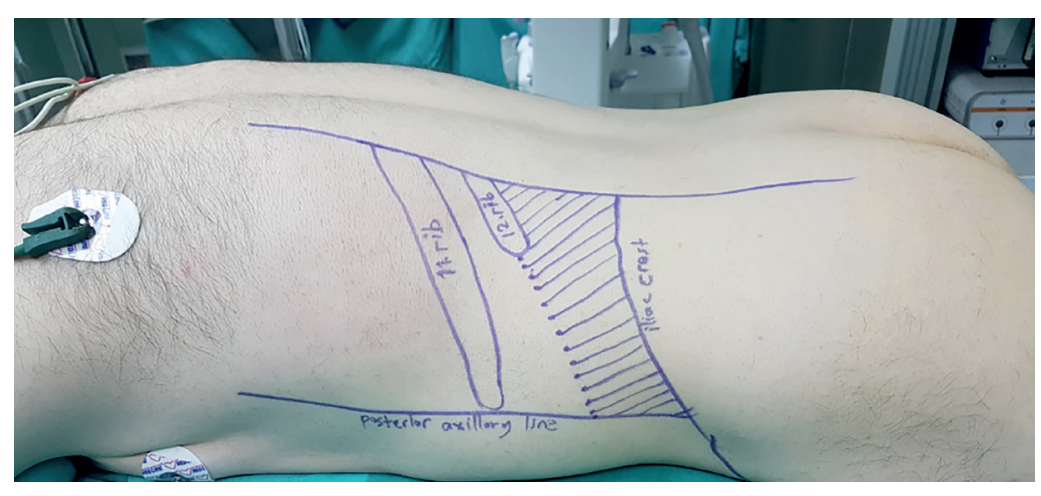

\section{Postoperative evaluation}

A complete blood count and renal function tests were obtained from all patients within 6 hours after the operation. In cases with a nephrostomy tube, the tube was removed on postoperative day 1 or 2 after antegrade nephrostography revealed ureteral drainage down to the bladder. The leakage longer than 48 hours was accepted as 'prolonged urine leakage' and JJ stent was placed following CT imaging. JJ stents were removed under local anesthesia. All patients were evaluated with renal function tests and a non-contrast spiral CT 1 month after the operation.

\section{Statistical analysis}

Data were analyzed by using Statistical Package for the Social Sciences software package version 20 (SPSS Inc., Chicago, IL, USA). Quantitative data were expressed as mean \pm std values on tables and categorical data were expressed with frequency (n) and percentages (\%). The distribution of the variables was measured by the Kolmogorov Smirnov test. Independent t test was used to compare independent groups. Pearson Correlation test was used to examine the relationship between variables. Pearson Chi-Square and Fisher Exact tests were used to compare the categorical data. The data were analyzed at 95\% confidence level and the threshold for statistical significance was accepted as $\mathrm{p}<0.05$ for all analyses.

\section{RESULTS}

Demographic data and stone characteristics are shown in Table-1. Age, sex, BMI, ASA score, stone size, stone localization and HN grade were similar between the groups because of fact that ' $1: 2$ matched pair' was performed.

Operative data are summarized in Table- 2 . The operation time and fluoroscopy time in supine $\mathrm{m}-\mathrm{PNL}$ was significantly shorter than prone $\mathrm{m}-\mathrm{PNL}$ group (58.1 \pm 45.9 vs. $80.1 \pm 40.0 \mathrm{~min}$ and $3.0 \pm 1.7 \mathrm{~min}$ vs. $4.9 \pm 4.5 \mathrm{~min}, \mathrm{p}=0.025$ and $\mathrm{p}=0.01$, respectively). While the rate of tubeless PNL was 37\% in supine $\mathrm{m}-\mathrm{PNL}$ group, it was $17.6 \%$ in prone $\mathrm{m}-\mathrm{PNL}$ group $(p=0.006)$. None of the patients had intercostal or upper pole access. There was no significant difference between the groups in terms of the intraoperative complications classified according to Satava.

Post-operative complications and outcomes are summarized in Table-3. When post-operative complications were compared according to the modified Clavien-Dindo classification, overall and subgroup complication rates were comparable between groups. Grade-4 complications (angioembolization and urosepsis) were observed in 3 patients in both groups. The hospitalization time was similar and there was no significant difference between the groups in terms of the success rates (supine m-PNL; 72.2\%, prone $\mathrm{m}-\mathrm{PNL} ; 71.3 \%, \mathrm{p}=0.902)$. When success was separately evaluated as single stone and multicaliceal stone, there was no significant difference. 
Table 1 - Demographic data and stone characteristics.

\begin{tabular}{|c|c|c|c|}
\hline & Supine $m$-PNL $(n=54)$ & Prone $m-P N L(n=108)$ & $p$ \\
\hline Sex (female/male) * & $15 / 39$ & $38 / 70$ & 0.343 \\
\hline Age (years)* & $43.4 \pm 11.9$ & $44.0 \pm 13.4$ & 0.813 \\
\hline BMI $\left(\mathbf{k g} / \mathbf{m}^{2}\right)^{*}$ & $27.3 \pm 3.9$ & $26.9 \pm 4.1$ & 0.609 \\
\hline ASA score* & $1.2 \pm 0.5$ & $1.1 \pm 0.4$ & 0.645 \\
\hline Previous ESWL / surgery & & & 0.553 \\
\hline ESWL & $9(16.7 \%)$ & $27(25.0 \%)$ & \\
\hline URS & $2(3.7 \%)$ & $6(5.6 \%)$ & \\
\hline PNL & $6(11.1 \%)$ & $16(14.8 \%)$ & \\
\hline Open Surgery & $3(5.6 \%)$ & $6(5.6 \%)$ & \\
\hline Stone opacity (opaque / non-opaque) & $50 / 4$ & $94 / 14$ & 0.289 \\
\hline Stone localization* & & & 0.821 \\
\hline Lower calyx & $8(14.8 \%)$ & $19(17.6 \%)$ & \\
\hline Middle calyx & 0 & 0 & \\
\hline Upper calyx & $2(3.7 \%)$ & $4(3.7 \%)$ & \\
\hline Pelvis & $23(42.6 \%)$ & $51(47.2 \%)$ & \\
\hline Multiple calyx & $21(38.9 \%)$ & $34(31.5 \%)$ & \\
\hline Stone size $(\mathrm{mm})^{*}$ & $25.8 \pm 7.9$ & $24.8 \pm 5.6$ & 0.886 \\
\hline Hydronephrosis (mild/severe)* & $43 / 11$ & $85 / 23$ & 0.891 \\
\hline
\end{tabular}

* Matching parameters (1:2 scenario)

\section{DISCUSSION}

In the literature, there is only one retrospective study comparing supine $\mathrm{m}-\mathrm{PNL}$ and prone m-PNL (16). In our study, supine m-PNL and prone $\mathrm{m}-\mathrm{PNL}$ were compared using ' $1: 2$ match pair analysis' in terms of success and complications. In the present study, general complication rates were similar in both group. Urosepsis was seen in one patient in the supine m-PNL group. Supin PNL provides the lower renal pelvic pressures. This is accepted as a protective factor for urosepsis (17). However, the presence of urosepsis in the supine m-PNL group suggested that the patient and operative factors (diabetes mellitus and long operation time) rather than surgical technique were effective in this patient. In PNL, the pleura and the colon are the most injured organs. In the literature, supine and prone PNL have different numbers for colon injury. In the supine position, intestines will be more anteriorly displaced and this condition will reduce the risk of colonic injury (18). In a comparative study using the CROES database, colon injury was found to be similar in both groups (3.4\% and 3.3\%, p=0.95) (19). However, in the randomized prospective studies, no colonic injuries have been reported in the supine PNL (19-21). In our study, no organ injuries were detected in both groups. In the supine m-PNL group, no upper pole access was performed due to positional difficulty in patients. Antegrade or retrograde flexible ureterorenoscope were used in cases where upper pole access was required. Samely, in the unique study comparing supine $\mathrm{m}$-PNL and prone $\mathrm{m}$-PNL in the literature, no upper pole access was performed in the supine group (16). 
Table 2 - Operative data.

\begin{tabular}{lccc}
\hline & Supine $\mathrm{m}-\mathrm{PNL}(\mathrm{n}=54)$ & Prone $\mathrm{m}-\mathrm{PNL}(\mathrm{n}=108)$ & $p$ \\
\hline Operation side (right / left) & $27 / 27$ & $51 / 57$ & 0.739 \\
Operation time $(\mathbf{m i n})$ & $58.1+45.9$ & $80.1+40.0$ & 0.025 \\
Fluoroscopy time (min) & $3.0 \pm 1.7$ & $4.9 \pm 4.5$ & 0.013 \\
Amplatz sheath size & & & 0.076 \\
$\quad 15 \mathrm{Fr}$ & $19(35.2 \%)$ & $21(19.4 \%)$ & \\
$16.5 \mathrm{Fr}$ & $20(37.0 \%)$ & $55(50.9 \%)$ & \\
$21 \mathrm{Fr}$ & $15(27.8 \%)$ & $32(29.6 \%)$ & 0.065 \\
Access & & & \\
$\quad$ Lower pole & $48(88.9 \%)$ & $85(78.7 \%)$ & $13(12.0 \%)$ \\
$\quad$ Middle pole & $6(11.1 \%)$ & $10(9.3 \%)$ & \\
$\quad$ Multiple access & 0 & $19(17.6 \%)$ & 0.006 \\
Tubuless procedure & $20(37.0 \%)$ & & 0.677 \\
Intraoperative complication & & $4(3.7 \%)$ & \\
$\quad$ Satava grade 1a & $2(3.7 \%)$ & $5(4.6 \%)$ & \\
$\quad$ Satava grade 2a & $1(1.9 \%)$ & & \\
\hline
\end{tabular}

In the meta-analysis including two randomized trials, it was reported that there was no statistically significant difference between supine standard PNL and prone standard PNL in terms of success rates $(83.5 \%$ vs. $81.6 \%$, respectively) (22). However, in another current meta-analysis, it was reported that prone standard PNL had significantly higher success rates than supine standard PNL (77.7\% vs. 74.4\%, $\mathrm{p}=0.0001)$. In the stu$\mathrm{dy}$, this difference was thought to be due to the fact that the nephroscope mobility was better in the prone PNL and that it was difficult to perform the upper pole access in the supine PNL (17). In the study comparing supine m-PNL and prone m-PNL, 54 and 126 patients were performed via supine $\mathrm{m}-\mathrm{PNL}$ and prone $\mathrm{m}-\mathrm{PNL}$; the stone-free rates were $74.1 \%$ and $76.2 \%$, respectively (16). Our study also confirmed that supine m-PNL and prone $\mathrm{m}$-PNL were not superior to each other in terms of success.
We concluded that prone PNL procedure has a longer operation time than supine PNL. This difference is due to the time for repositioning the patient in prone PNL. In the meta-analysis study, it was reported that supine standard PNL had the advantage of an average operation time of $18 \mathrm{~min}$ and this difference was statistically significant (17). This result was also confirmed by a prospective randomized trial (21). In the study comparing supine $\mathrm{m}-\mathrm{PNL}$ and prone $\mathrm{m}-\mathrm{PNL}$, operation times were $55 \mathrm{~min}$ and $82 \mathrm{~min}$ in supine $\mathrm{m}-\mathrm{PNL}$ and prone $\mathrm{m}-\mathrm{PNL}$, respectively (16). In our study, the difference in operation time between supine and prone m-PNL was of average 22 minutes.

Because of the fact that it has some advantages in terms of cardiovascular, respiratory and anesthesia application, supine is a more accepted position by anesthetists than prone. There is a risk of the endotracheal tube being removed 
Table 3 - Postoperative complications and outcomes.

\begin{tabular}{lccc}
\hline & Supine m-PNL (n=54) & Prone m-PNL (n=108) & $p$ \\
\hline Clavien - Dindo classification & & & 0.452 \\
$\quad$ Grade 0 & $38(70.4 \%)$ & $76(70.4 \%)$ & \\
$\quad$ Grade 1 & $5(9.3 \%)$ & $17(15.7 \%)$ & \\
$\quad$ Grade 2 & $1(1.9 \%)$ & $5(4.6 \%)$ & \\
$\quad$ Grade 3a & $2(3.7 \%)$ & $3(2.8 \%)$ & \\
$\quad$ Grade 3b & $5(9.3 \%)$ & $4(3.7 \%)$ & 0.521 \\
$\quad 3$ Grade 4 & $3(5.6 \%)$ & $3(2.8 \%)$ & 0.817 \\
Double-J stent placement & $1(1.9 \%)$ & $4(3.7 \%)$ & 0.376 \\
Fever & $3(5.6 \%)$ & $7(6.5 \%)$ & 0.644 \\
Hematocrit drop (gr/dL) & $3.9 \pm 3.3$ & $3.2 \pm 3.0$ & 0.216 \\
Transfusion & $4(7.4 \%)$ & $6(5.6 \%)$ & 0.214 \\
Angioembolization & $2(3.7 \%)$ & $1(0.9 \%)$ & 0.401 \\
Urosepsis & $1(1.9 \%)$ & $2(1.8 \%)$ & 0.902 \\
Hospitalization time (hour) & $56.3 \pm 62.5$ & $66.0 \pm 37.2$ & 0.605 \\
Overall success & $39(72.2 \%)$ & $77(71.3 \%)$ & 0.391 \\
$\quad$ Success in isolated calyx stones & $22(66.7 \%)$ & $53(71.6 \%)$ & $24(70.6 \%)$ \\
$\quad$ Success in multiple calyx stones & $17(81.0 \%)$ &
\end{tabular}

during the positioning to prone and the possibility of intervention to airway becomes limited after the patient is positioned. Furthermore, in the prone position, the risks of nerve tension, musculoskeletal injuries and visual impairment due to increased ocular pressure are more likely $(23,24)$. These risks are clinically insignificant in patients at low risk (ASA 1/2) groups (25). Despite its significant disadvantages, the prone position is used more often by surgeons. The reason for this is that surgeons are more accustomed to prone position. In the supine position, the surgeon can comfortably sit during the operation, and $\mathrm{x}$-ray exposure is reduced because puncture and dilation of the nephrostomy tract are quite perpendicular to the body and the operator's hands are outside the fluoroscopic field. Furthermore, by rotating the legs into the lithotomy position, combined antegrade and retrograde procedures can effectively be performed in the supine position. This represents the main advantage of this procedure because it combines the benefits of percutaneous and ureteroscopic intrarenal surgery in selected cases of contemporary treatment of bilateral stones (26).

Although the present study is a 1:2 match pair analysis study, it has some limitations. The main limitations of the present study is its retrospective nature and the relatively small sample size. Thus, large-scale randomized trials should be encouraged to be designed, so that the above conclusions can be verified with an increased statistical power. Secondly, we did not match stone compositions for comparison. Theoretically, SFR could be affected by the differences in stone components between the two groups. Thirdly, we excluded the patients with skeletal malformations and with kid- 
ney stones located in the upper pole. As surface area used in prone $\mathrm{m}-\mathrm{PNL}$ is extended, performing an access to the upper calyx is easier than supine m-PNL. In patients with wide hips and thin calices, it can be more difficult or even impossible to reach the upper calyx with a rigid nephroscope in supine position. So, the patients with upper pole stones were excluded from the study. Stone treatment in patients with skeletal deformity can be a serious problem for urologists. Skeletal deformities make both conventional and minimal invasive surgical interventions difficult. In these patients, it may be necessary to perform stone treatment by giving different positions other than supine or prone position. Also, for these patients, PNL may not always be the appropriate option. Instead, open surgery, laparoscopic-assisted PNL or f-URS may be more suitable options. Because of these reasons, the patients with skeletal deformity were excluded from our study. Another limitation of our study is that Guy's stone score is not included. This system includes some parameters such as the presence of upper pole stone, anatomical abnormalities (calyceal diverticulum) and skeletal deformities (spina bifida, spinal injury). So, we were unable to use the Guy's score in the present study.

\section{CONCLUSIONS}

In the treatment of lower pole, middle pole and renal pelvic stones, supine m-PNL and prone $\mathrm{m}$-PNL procedures have similar success rates. There is no significant difference in terms of general complication rates. However, supine PNL is more advantageous in terms of operation and fluoroscopy times.

\section{CONFLICT OF INTEREST}

None declared.

\section{REFERENCES}

1. Türk C, Petř́k A, Sarica K, Seitz C, Skolarikos A, Straub $M$, et al. EAU Guidelines on Diagnosis and Conservative Management of Urolithiasis. Eur Urol 2016;69:468-74.

2. Jackman SV, Docimo SG, Cadeddu JA, Bishoff JT, Kavoussi $L R$, Jarrett TW. The "mini-perc" technique: a less invasive alternative to percutaneous nephrolithotomy. World J Urol. 1998;16:371-4.
3. Desai J, Solanki R. Ultra-mini percutaneous nephrolithotomy (UMP): one more armamentarium. BJU Int. 2013;112:10469.

4. Zeng G, Wan S, Zhao Z, Zhu J, Tuerxun A, Song C, et al. Super-mini percutaneous nephrolithotomy (SMP): a new concept in technique and instrumentation. BJU Int. 2016;117:655-61.

5. Desai MR, Sharma R, Mishra S, Sabnis RB, Stief C, Bader M. Single-step percutaneous nephrolithotomy (microperc): the initial clinical report. J Urol. 2011;186:140-5.

6. Ruhayel Y, Tepeler A, Dabestani S, MacLennan S, Pet ík A, Sarica K, et al. Tract Sizes in Miniaturized Percutaneous Nephrolithotomy: A Systematic Review from the European Association of Urology Urolithiasis Guidelines Panel. Eur Urol. 2017;72:220-35.

7. Cracco CM, Scoffone CM. ECIRS (Endoscopic Combined Intrarenal Surgery) in the Galdakao-modified supine Valdivia position: a new life for percutaneous surgery? World J Urol. 2011;29:821-7.

8. Kerbl K, Clayman RV, Chandhoke PS, Urban DA, De Leo BC, Carbone JM. Percutaneous stone removal with the patient in a flank position. J Urol. 1994;151:686-8.

9. Lehman T, Bagley DH. Reverse lithotomy: modified prone position for simultaneous nephroscopic and ureteroscopic procedures in women. Urology. 1988;32:529-31.

10. Papatsoris AG, Zaman F, Panah A, Masood J, El-Husseiny T, Buchholz N. Simultaneous anterograde and retrograde endourologic access: "the Barts technique". J Endourol. 2008;22:2665-6.

11. Valdivia Uría JG, Lachares Santamaría E, Villarroya Rodríguez S, Taberner Llop J, Abril Baquero G, Aranda Lassa JM. [Percutaneous nephrolithectomy: simplified technic (preliminary report)]. Arch Esp Urol. 1987;40:177-80.

12. Valdivia Uría JG, Valle Gerhold J, López López JA, Villarroya Rodriguez S, Ambroj Navarro C, Ramirez Fabián M, et al. Technique and complications of percutaneous nephroscopy: experience with 557 patients in the supine position. J Urol. 1998;160(6 Pt 1):1975-8.

13. Scoffone CM, Cracco CM. Invited review: the tale of ECIRS (Endoscopic Combined IntraRenal Surgery) in the Galdakaomodified supine Valdivia position. Urolithiasis. 2018;46:11523.

14. Satava RM. Identification and reduction of surgical error using simulation. Minim Invasive Ther Allied Technol. 2005;14:257-61.

15. Dindo D, Demartines N, Clavien PA. Classification of surgical complications: a new proposal with evaluation in a cohort of 6336 patients and results of a survey. Ann Surg. 2004;240:205-13. 
16. Tokatlı Z, Gokce MI, Süer E, Sa lam R. Supine or prone position for mini-PNL procedure: does it matter. Urolithiasis. 2015;43:261-4.

17. Yuan D, Liu Y, Rao H, Cheng T, Sun Z, Wang Y, et al. Supine Versus Prone Position in Percutaneous Nephrolithotomy for Kidney Calculi: A Meta-Analysis. J Endourol. 2016;30:754-63.

18. Boon JM, Shinners B, Meiring JH. Variations of the position of the colon as applied to percutaneous nephrostomy. Surg Radiol Anat. 2001;23:421-5.

19. Valdivia JG, Scarpa RM, Duvdevani M, Gross AJ, Nadler RB, Nutahara K, et al. Supine versus prone position during percutaneous nephrolithotomy: a report from the clinical research office of the endourological society percutaneous nephrolithotomy global study. J Endourol. 2011;25:1619-25.

20. Duty B, Okhunov Z, Smith A, Okeke Z. The debate over percutaneous nephrolithotomy positioning: a comprehensive review. J Urol. 2011;186:20-5.

21. De Sio M, Autorino R, Quarto G, Calabrò F, Damiano R, Giugliano $F$, et al. Modified supine versus prone position in percutaneous nephrolithotomy for renal stones treatable with a single percutaneous access: a prospective randomized trial. Eur Urol. 2008;54:196-202.

22. Liu L, Zheng $S, X u Y$, Wei Q. Systematic review and metaanalysis of percutaneous nephrolithotomy for patients in the supine versus prone position. J Endourol. 2010;24:1941-6.
23. Edgcombe $\mathrm{H}$, Carter $\mathrm{K}$, Yarrow $\mathrm{S}$. Anaesthesia in the prone position. Br J Anaesth. 2008;100:165-83.

24. Patel RM, Okhunov Z, Clayman RV, Landman J. Prone Versus Supine Percutaneous Nephrolithotomy: What Is Your Position? Curr Urol Rep. 2017;18:26.

25. Al-Dessoukey AA, Moussa AS, Abdelbary AM, Zayed A, Abdallah R, Elderwy $A A$, et al. Percutaneous nephrolithotomy in the oblique supine lithotomy position and prone position: a comparative study. J Endourol. 2014;28:1058-63.

26. Giusti G, Proietti S, Pasin L, Casiraghi GM, Gadda GM, Rosso M, et al. Simultaneous Bilateral Endoscopic Manipulation for Bilateral Renal Stones. Urology. 2016;94:265-9.

\section{Correspondence address:}

Akif Erbin, MD

Department of Urology,

Haseki Traning and Research Hospital, Istanbul,

Turkey

Phone: + 905065431062

Fax: + 902125294400

E-mail: akiferbin@hotmail.com 